\section{Uma proposta metodológica para a elaboração de revisões clínicas}

\author{
A methodological proposal for the preparation of clinical reviews \\ Una propuesta metodológica para la elaboración de revisiones \\ clínicas
}

\section{Resumo}

As revisões clínicas são sumários de evidências com escopo amplo e orientação à prática profissional, ocupando um dos mais altos níveis da pirâmide da assistência à saúde baseada em evidências. Com base nessa pirâmide, refletimos sobre as instruções aos autores de periódicos em medicina de família e comunidade para propor instruções para a escrita de revisões clínicas. As instruções abrangem a busca, avaliação, síntese e análise das evidências clínicas, além do planejamento e redação da revisão. Esperamos que estas instruções ajudem a elevar a quantidade e qualidade das revisões clínicas em medicina de família e comunidade no país.

Palavras-chave: Literatura de Revisão como Assunto; Metodologia; Medicina de Família e Comunidade; Medicina Baseada em Evidências

\begin{abstract}
Clinical reviews are evidence summaries with broad scope and orientation to professional practice, occupying one of the highest levels in the pyramid of evidence-based health care. Based on this pyramid, we reflect on instructions to the authors of journals in family medicine to present instructions on how to write clinical reviews. The instructions cover how to search, appraise, synthesize and analyze clinical evidence, and how to plan and report the review. We hope these instructions will help improve the quantity and quality of clinical reviews in family medicine in Brazil.
\end{abstract}

Keywords: Review Literature as Topic; Methodology; Family Practice; Evidence-Based Medicine

\section{Resumen}

Revisiones clínicas son resúmenes de evidencia con un amplio alcance y orientación a la práctica profesional, ocupando uno de los niveles más altos en la pirámide de la asistencia sanitaria basada en pruebas. Con base en esta pirámide, reflexionamos sobre las instrucciones de los autores de las revistas de medicina familiar para presentar instrucciones sobre cómo escribir revisiones clínicas. Las instrucciones cubren cómo buscar, evaluar, sintetizar y analizar evidencia clínica, y cómo planificar e reportar la revisión. Esperamos que estas instrucciones ayuden a mejorar la cantidad y calidad de las revisiones clínicas en medicina familiar y comunitaria en Brasil.

Palabras clave: Literatura de Revisión como Asunto; Metodología; Medicina Familiar y Comunitaria; Medicina Basada en la Evidencia

Como citar: Fontenelle LF, Brandão DJ. Uma proposta metodológica para a elaboração de revisões clínicas. Rev Bras Med Fam Comunidade. 2018;13(40):1-10. http://dx.doi.org/10.5712/rbmfc13(40)1871
Leonardo Ferreira Fontenelle

Diego José Brandão

Universidade Vila Velha (UVV).

Vila Velha, ES, Brasil.

leonardof@leonardof.med.br

(Autor correspondente);

diegojbrandao@yahoo.com.br
Fonte de financiamento:

declaram não haver.

Parecer CEP:

não se aplica.

Conflito de interesses:

Leonardo Ferreira Fontenelle tornou-se coeditor da Revista Brasileira de Medicina de Família e Comunidade antes de esta proposta ter sido aprovada para publicação; e é preceptor de um programa de residência médica em medicina de família e comunidade onde se propõe que os trabalhos de conclusão de curso sejam revisões clínicas. Diego José Brandão é supervisor de um programa de residência médica em medicina de família e comunidade onde se propõe que os trabalhos de conclusão de curso sejam revisões clínicas.

Procedência e revisão por pares: revisado por pares.

Recebido em: 03/09/2018.

Aprovado em: 03/10/2018. 


\section{Introdução}

Praticar medicina baseada em evidências significa resolver situações encontradas na prática clínica aliando a melhor evidência disponível ao julgamento profissional e à preferência do paciente..$^{1-3}$ Infelizmente, a falta de tempo e habilidade de grande parte dos médicos limita a efetividade da análise crítica de artigos enquanto estratégia para a transformação da prática clínica. ${ }^{2-6}$

A "pirâmide 5.0 da assistência à saúde baseada em evidências" é um modelo para hierarquizar as fontes de informação conforme sua eficiência e utilidade para os profissionais envolvidas na assistência à saúde. ${ }^{7}$ Quando um profissional de saúde tem uma dúvida, deve consultar primeiramente os níveis superiores da pirâmide, consultando consecutivamente os níveis inferiores, caso necessário. ${ }^{7}$ Uma estratégia ainda mais ágil é utilizar ferramentas de busca federada, como ACCESSSS e Trip, que retornam simultaneamente resultados para múltiplos níveis da pirâmide. ${ }^{7}$

O topo da pirâmide, "sistemas", seriam sistemas computadorizados de suporte à decisão, integrados a prontuários eletrônicos para que o profissional nem ao menos precisasse procurar pelas evidências ou recomendações. ${ }^{7}$ Esse tipo de ferramenta para a medicina baseada em evidências continua sendo a menos encontrada, embora algum progresso tenha sido feito nesse sentido. ${ }^{7}$

O próximo nível mais eficiente da pirâmide são os "sumários sintetizados para referência clínica", que integram diretrizes, revisões sistemáticas e estudos originais em um conteúdo direcionado às necessidades de consulta da prática clínica. ${ }^{7} \mathrm{O}$ protótipo desse nível são textos eletrônicos continuamente atualizados, como DynaMed Plus, UpToDate e BMJ Best Practice. ${ }^{7}$

Os próximos níveis são as diretrizes, as revisões sistemáticas e os estudos originais, consecutivamente. ${ }^{7}$ Diretrizes são recomendações derivadas sistematicamente, que integram a melhor evidência, o julgamento profissional e a preferência do paciente; ${ }^{7,8}$ revisões sistemáticas consistem na busca, avaliação e síntese sistemática da evidência disponível sobre questões bem definidas; ${ }^{2,7,9}$ e estudos originais dispensam apresentação. Dentro de cada um desses três níveis da base da pirâmide, profissionais de saúde devem lançar mão preferencialmente de seleções filtradas manualmente, resumidas e avaliadas por terceiros, como no McMaster PLUS, ACP Journal Club ou os "departamentos" da revista American Family Physician. 7,10

Revisões clínicas são uma forma popular de atualização clínica, articulando a experiência dos autores ao estado da literatura científica sobre o tema em questão. ${ }^{11}$ A redação concisa e orientada à prática profissional posiciona os artigos de revisão clínica como uma forma de sumário, apesar da falta de uma atualização contínua.

Muitas revistas focadas em medicina de família e comunidade, além de revistas médicas gerais como The BMJ e New England Journal of Medicine, reservam um espaço para revisões clínicas. Apresentamos a seguir uma proposta de como o médico de família e comunidade pode elaborar uma revisão clínica com vistas à publicação na forma de artigo em periódico, com ênfase na Revista Brasileira de Medicina de Família e Comunidade.

\section{Notas metodológicas}

Esta proposta foi construída por meio da reflexão sobre as instruções aos autores de revistas voltadas para médicos de família e comunidade. Para serem incluídas, as revistas deveriam estar em atividade, 
serem revisadas por pares, e fornecerem instruções aos autores específicas para revisões clínicas. Não foram incluídos anais de congressos, boletins ou revistas que não disponibilizassem as informações em sua página da World Wide Web em português, inglês ou espanhol. As revistas foram identificadas a partir do Portal de Revistas Científicas em Ciências da Saúde (Biblioteca Virtual em Saúde - BVS), em julho de 2018, utilizando o descritor medicina de família e comunidade ou os termos family, community ou general.

Foram encontradas dez revistas de medicina de família e comunidade atendendo aos critérios de inclusão (Quadro 1). Essas revistas são publicadas por dez diferentes organizações, em sete países e quatro idiomas (uma revista é bilíngue, sendo publicada também em francês). As revistas variaram quanto ao volume de revisões clínicas publicadas anualmente, foco esperado das revisões, número de tipos diferentes de revisão clínica e detalhamento das instruções. As revistas que mais contribuíram para esta proposta foram American Family Physician (AFP), Canadian Family Physician (CFP), Formación Médica Continuada en Atención Primaria (FMC), Journal of Family Practice (JFP), Journal of the American Board of Family Medicine (JABFM), Revista Brasileira de Medicina de Família e Comunidade (RBMFC) e Revista Valenciana de Medicina de Familia (FML).

Quadro 1. Revistas incluídas no desenvolvimento da proposta.

\begin{tabular}{|ll|}
\hline Revista & Endereço \\
\hline American Family Physician (AFP) & http://www.aafp.org/journals/afp.html \\
Australian Family Physician & http://www.racgp.org.au/afp \\
British Journal of General Practice & http://bjgp.org \\
Canadian Family Physician (CFP) & http://www.cfp.ca \\
Formación Médica Continuada En Atención Primaria (FMC) & http://www.fmc.es \\
Hong Kong Practitioner & http://www.hkcfp.org.hk/pages_121_104.html \\
Journal of Family Practice (JFP) & $\mathrm{http://www.mdedge.com/jfponline}$ \\
Journal of the American Board of Family Medicine (JABFM) & $\mathrm{http://www.jabfm.org}$ \\
Revista Brasileira de Medicina de Família e Comunidade (RBMFC) & $\mathrm{http://www.rbmfc.org.br}$ \\
Revista Valenciana de Medicina de Familia (FML) & $\mathrm{http://www.revistafml.es}$ \\
\hline
\end{tabular}

O conteúdo das instruções (e as reflexões nele baseadas) foi organizado utilizando o modelo SALSA ("buSca, AvaLiação, Síntese e Análise"), ${ }^{9}$ acrescentando-se ainda uma seção sobre o planejamento e outra sobre a redação da revisão clínica. A reflexão sobre as instruções aos autores teve como referencial a "pirâmide 5.0 da assistência à saúde baseada em evidências". ${ }^{7}$

\section{Planejando a revisão clínica}

Quando se começa a escrever um artigo, é boa prática ter em mente a revista para a qual ele será enviado. No caso de revisões clínicas, isso é ainda mais importante, pois algumas revistas recomendam a submissão de uma proposta antes de a revisão ser iniciada, e as revistas FMC e FML aceitam apenas as revisões clínicas que encomendam. Além disso, uma revista (AFP) não aceita artigos de autores com conflitos de interesse.

Conforme recomendado pela revista com as mais detalhadas instruções aos autores (AFP), antes de buscar evidências é bom escrever uma lista de questões-chave e controvérsias clínicas, para então respondê-las o melhor possível. Em termos de um projeto de pesquisa, se o objetivo geral é revisar as 
evidências clínicas para a abordagem da doença hepática gordurosa não alcoólica na atenção primária à saúde, um objetivo específico poderia ser identificar se e quando é necessário encaminhar para biópsia hepática a fim de verificar a presença de esteatohepatite.

Para escolher as questões-chave, é necessário aliar experiência clínica a um estudo prévio da condição clínica ("do que se trata") e sua epidemiologia. Em geral, esse estudo prévio serve de base para a introdução da revisão clínica. Outra consideração é que essa lista de questões-chave deve ser tão detalhada quanto possível, para facilitar a síntese preliminar das evidências (ver seção "Sintetizando as evidências").

\section{Buscando as evidências}

Ao contrário da maioria dos tipos de revisão de literatura, ${ }^{9}$ as revisões clínicas incluem preferencialmente revisões sistemáticas e diretrizes clínicas. As pesquisas originais são utilizadas apenas quando alguma questão-chave não pode ser adequadamente respondida com os níveis superiores da pirâmide. Por outro lado, sendo um sumário das evidências clínicas disponíveis, as revisões clínicas não devem utilizar outros sumários como referência.

Diretrizes clínicas e revisões sistemáticas podem ser buscadas com o filtro "systematic reviews" do PubMed Clinical Queries ou com o tipo de estudo "revisão sistemática” do índice LILACS (Quadro 2). Revisões produzidas pela Cochrane Collaboration podem ser encontradas tanto através do PubMed Clinical Queries quanto na própria Cochrane Library. Infelizmente, a National Guidelines Clearinghouse e a Database of Abstracts of Reviews of Effects (DARE) deixaram de ser atualizadas, respectivamente, pelo governo dos Estados Unidos da América e pelo Centre for Reviews and Dissemination. Quando necessário, artigos originais podem ser encontrados através dos filtros "clinical study categories" da PubMed Clinical Queries ou os "tipos de estudo" correspondentes da LILACS.

Quadro 2. Recursos para a busca de evidências clínicas.

\begin{tabular}{|ll|}
\hline Recurso & Endereço \\
\hline ACCESSSS Federated Search & https://www.accessss.org \\
ACP Journal Club & https://annals.org/aim/journal-club \\
BMJ Best Practice & https://brasil.bestpractice.bmj.com \\
Cochrane Library & http://www.cochranelibrary.com \\
DynaMed PLUS & http://psbe.ufrn.br \\
Epistemonikos & https://www.epistemonikos.org \\
Evidence-Based Medicine Guidelines & http://www.ebm-guidelines.com \\
Guidelines International Network & https://www.g-i-n.net \\
LILACS & http://lilacs.bvsalud.org \\
McMaster PLUS & https://www.accessss.org \\
PubMed Clinical Queries & https://www.ncbi.nlm.nih.gov/pubmed/clinical \\
Trip & https://www.tripdatabase.com \\
UpToDate & https://www.uptodate.com \\
\hline
\end{tabular}


Na prática, é mais vantajoso usar buscas federadas como ACCESSSS e Trip (Quadro 2), que permitem a busca simultânea em várias bases de dados (exceto LILACS). No caso de artigos originais, revisões sistemáticas e diretrizes clínicas, essa busca abrange a seleção filtrada manualmente, resumida e avaliada de McMaster PLUS, ACP Journal Club e Evidence-Based Medicine Guidelines, além da seleção filtrada automaticamente de PubMed Clinical Queries. A ACCESSSS busca ainda os sumários clínicos DynaMed PLUS, UpToDate e Best Practice.

A estratégia de busca é geralmente simples, consistindo em palavras-chave e termos correlatos ao tema da revisão clínica, como “(depression OR 'feeling depressed') OR 'depressive disorder'”. Dentre os recursos listados no Quadro 2, LILACS e PubMed Clinical Queries são os únicos a contar com palavraschave padronizadas, chamados respectivamente DeCS (Descritores em Ciências da Saúde) e MeSH (Medical Subject Headings). Caso o número de resultados seja excessivo, pode-se restringir a busca acrescentando termos como "primary health care" e "family practice".

A mesma estratégia de busca pode ser utilizada para encontrar artigos originais, utilizando filtros apropriados na PubMed Clinical Queries, LILACS ou ACCESSSS, caso as revisões sistemáticas e diretrizes clínicas não bastem para responder às questões-chave. Para evitar um número excessivo de artigos, a estratégia de busca pode receber termos relacionados à atenção primária à saúde e/ou medicina de família e comunidade, ou implementar a estratégia PICOS (pacientes, intervenção, comparação, desfecho, tipo de estudo $)^{12}$ no caso de evidências sobre intervenções. A AFP recomenda ainda uma outra forma de complementar a busca por evidências: incluindo artigos originais citados por sumários como DynaMed, BMJ Best Practice ou UpToDate (Quadro 2); o Clinical Evidence foi descontinuado.

Ao contrário das revisões sistemáticas, as revisões de literatura não precisam - nem devem - incluir todas as evidências clínicas aplicáveis. Basta incluir as diretrizes clínicas e revisões sistemáticas mais recentes, abrangentes e relevantes. De uma forma geral, referências publicadas há mais de 10 anos só devem ser utilizadas em casos especiais. Com relação à relevância, devem ser enfatizados estudos realizados na atenção primária à saúde e/ou que abordaram desfechos clinicamente relevantes, como recomendado pela RBMFC.

Ao fim da busca por evidências, o autor da revisão clínica tem uma lista de referências, que podem ser organizadas com um editor de textos (como Word ou LibreOffice), bloco de notas (como Evernote, Keep ou SimpleNote) ou gerenciador de referências bibliográficas (como EndNote, Mendeley ou Zotero). Não é necessário registrar quais, ou quantas, referências deixaram de ser incluídas.

\section{Avaliando as evidências}

A robustez de uma revisão depende não apenas de seus métodos, mas também da robustez dos estudos nela incluídos. Por isso, algumas revistas dos Estados Unidos (AFP, JFP, JABFM) orientam os autores de revisões clínicas a avaliar a força das recomendações utilizando a Strength of Recommendation Taxonomy (SORT). ${ }^{13}$ A CFP deixou a SORT em favor de uma classificação, ainda mais simples, descrita em suas instruções aos autores. ARBMFC não exige a avaliação das evidências, mas instrui os autores a preferir evidências sobre desfechos clínicos relevantes, o que acaba melhorando a força das recomendações. 
A SORT foi desenvolvida especificamente para graduar a força das recomendações-chave (diagnósticas, prognósticas ou de tratamento/prevenção/rastreamento) em revisões clínicas em medicina de família e comunidade. A força de uma recomendação é baseada na qualidade dos estudos individuais e na consistência entre os estudos que dão suporte a cada recomendação. A qualidade dos estudos individuais é avaliada pelo tipo de delineamento (por exemplo: revisão sistemática, ensaio controlado randomizado, série de casos), entre outras características do estudo. O fato de um estudo não avaliar desfechos orientados ao paciente (apenas orientados à doença, ou de ciência básica) força sua classificação com a pior qualidade. ${ }^{13}$

Caso a força das recomendações seja avaliada apenas para as principais recomendações, como instruído pela AFP, a avaliação das evidências pode ser feita entre as etapas de síntese e análise das evidências. Por outro lado, caso a revisão clínica avalie todas as evidências citadas, pode ser mais produtivo avaliar a qualidade dos estudos individuais antes de ou durante a síntese das evidências, para então avaliar a avaliação da consistência entre os estudos (para cada recomendação-chave) e chegar à força das recomendações durante a análise das evidências (ver mais adiante).

A SORT deve ser diferenciada do sistema Grading of Recommendations Assessment, Development and Evaluation (GRADE), com ferramentas para a avaliação da qualidade da evidência, graduação das recomendações e tomada de decisão. ${ }^{2,14-18}$ O GRADE se tornou o padrão de fato para revisões sistemáticas e diretrizes clínicas, ${ }^{2}$ mas ainda não está claro como seria utilizado para a elaboração revisões clínicas. Talvez a adoção do GRADE pelo UpToDate possa iluminar a forma de adoção do GRADE em revisões clínicas. ${ }^{19}$

\section{Sintetizando as evidências}

Assim como em outros tipos de revisão, a elaboração de uma revisão clínica passa pela síntese das milhares de palavras das evidências incluídas. Essa síntese não é incluída no artigo final, e não é mencionada nas instruções aos autores das revistas consultadas. A síntese proposta a seguir é uma forma de organização para as etapas seguintes, evitando o imobilismo que pode seguir-se à busca de evidências.

A síntese das evidências clínicas é uma forma de fichamento: as referências obtidas na etapa de busca já foram registradas, agora se registram as principais conclusões. Quem imprime os artigos pode preferir fazer anotações ou destacar trechos nos artigos, mas para quem trabalha diretamente no computador a estratégia mais eficiente é organizar as conclusões por questão-chave.

Começa-se com um documento eletrônico em branco (num editor de texto ou bloco de notas), documento esse que é preenchido com as seções do manuscrito como deverá ser enviado à revista. Dentro de cada seção, coloca-se um subtítulo para cada questão-chave; e cada conclusão é colocada sob a questão-chave correspondente. Não é necessário anotar a referência completa para cada conclusão registrada: basta citar autor e ano, para o autor da revisão identificar a referência na lista de referências elaborada durante a etapa de busca por evidências.

O Quadro 3 ilustra parcialmente essa síntese de evidências, incluindo citações para uma lista de referências organizada à parte, e ainda uma avaliação da qualidade individual dos artigos. 
Quadro 3. Exemplo parcial de síntese preliminar.

- Avaliação

- Como diferenciar a cólica renal de outras dores abdominais?

- Os resultados claramente mostram que dor abdominal aguda com apetite normal, curta duração da dor ( $\leq 12$ h), dor à percussão lombar ou renal e hematúria (> 10 eritrócitos) são indicativos de cólica renal aguda [...]. Adicionalmente, a possibilidade de pielonefrite obstrutiva em combinação com cólica renal deve ser considerada clinicamente. [Eskelinen et al., 1998 - SORT 2]

- O escore STONE pode agregar pacientes em grupos de baixo, médio e alto risco, e prediz a litíase ureteral com mais especificidade do que a intuição médica. No entanto, em sua presente forma, o escore STONE carece de acurácia suficiente para permitir aos clínicos deixar de solicitar tomografia computadorizada para um cálculo ureteral suspeito.[Wang el al., 2016 - SORT 2 (o STONE original foi validado, mas sua modificação, não)]

- Recomendações

- Como fazer a analgesia inicial da cólica renal aguda?

- Os anti-inflamatórios não esteroides (AINE) são um tratamento efetivo para a cólica renal quando comparados a placebo ou antiespasmódicos. A adição de antiespasmódicos aos AINE não resulta em melhor controle da dor. Dados sobre outras medicações não opioides, não AINE são escassos. Não foram relatados na literatura efeitos adversos maiores pelo uso de AINE para o tratamento da cólica renal. [Afshar et al., 2015 - SORT 2]

- Qual é o papel da terapia expulsiva no manejo conservador da cólica renal?

- Houve evidência de moderada qualidade de que bloqueadores alfa facilitam a passagem de cálculos ureterais. [...] Não parece haver benefício para o tratamento com bloqueadores alfa entre pacientes com cálculos ureterais pequenos. Pacientes com cálculos maiores tratados com bloqueadores alfa, no entanto, têm um risco $57 \%$ de expelir o cálculo em comparação aos controles. O efeito dos bloqueadores alfa foi independente da localização do cálculo. Em comparação aos controles, pacientes que receberam bloqueadores alfa tiveram tempos significativamente mais curtos até a passagem do cálculo (evidência de moderada qualidade), menos episódios de dor (evidência de baixa qualidade), menor risco de intervenções cirúrgicas (evidência de moderada qualidade), e menor risco de admissão ao hospital (evidência de moderada qualidade). O risco de um evento adverso sério foi semelhante entre os de grupos tratamento e controle (evidência de baixa qualidade). [Hollingsworth et al., 2016 - SORT 2]

\section{Analisando as evidências}

O objetivo de uma revisão clínica é informar a prática profissional, e não apenas sistematizar a literatura científica. Ao elaborar uma revisão clínica, o autor deve ter em mente a sequência das informações a serem coletadas na consulta e das decisões a serem tomadas, e utilizar seu discernimento profissional para contextualizar as evidências clínicas nessa sequência.

Esse discernimento profissional transparece ao longo de todo o artigo, e algumas revistas (AFP, JFP, RBMFC) recomendam especificamente que os autores apresentem seus comentários ao longo da apresentação das evidências clínicas. É uma situação análoga à de muitos artigos de pesquisa qualitativa, que discutem os resultados na medida em que os vão relatando.

A análise das evidências clínicas é incorporada não apenas no texto, mas também nas ilustrações. A RBMFC, por exemplo, espera dos autores que forneçam para a seção de avaliação um fluxograma, e para a seção de recomendações um quadro com as principais recomendações e as evidências que lhe dão suporte. Na AFP, a força das recomendações é apresentada no quadro com as principais recomendações, além de ao longo do texto. 


\section{Redigindo a revisão clínica}

Cada revista fornece instruções específicas sobre como apresentar a revisão clínica. As revistas variam com relação ao limite de números de palavras, conjunto de seções e tipo de ilustrações. ARBMFC, por exemplo, tem um limite de 2.500 palavras; exige as seções Introdução, Métodos e Considerações finais; e sugere tabelas, gráficos e fluxogramas em certas seções.

Como outros tipos de artigo, a revisão clínica não precisa ser escrita de forma linear. Mesmo assim, pode ser que a introdução já esteja quase pronta desde o começo, graças à recapitulação da patologia e da epidemiologia realizada para escolher as questões-chave. A seguir, se descreve a estratégia de busca, que também pode ser aproveitada em grande do que se escreveu durante o planejamento da revisão clínica. As seções seguintes podem ser escritas mais ou menos em qualquer ordem, e as ilustrações (quadros, fluxogramas...) podem ser preparadas antes ou depois do texto propriamente dito. A discussão ou conclusão, quando exigida pela revista, deve ser a última ou uma das últimas partes a ser escrita.

Cumpre destacar que a própria elaboração de uma revisão clínica (ou qualquer tipo de artigo) não é tão linear quanto possa parecer. A escolha do tema se dá mais ou menos ao mesmo tempo que a escolha da revista, e pode ser reconsiderada após a busca das evidências. Da mesma forma, as questões-chave correm o risco de serem revistas após a síntese ou a análise das evidências. Embora a busca por revisões sistemáticas e diretrizes clínicas seja feita de uma só vez, a síntese das evidências pode identificar lacunas a serem preenchidas com buscas por pesquisas originais, focadas em questões específicas. Por fim, a análise das evidências é frequentemente entrelaçada com a redação do artigo, pois os resultados da análise precisarão ser registrados de alguma forma, e a redação do artigo frequentemente força o autor a pensar mais claramente sobre o assunto.

\section{Considerações finais}

As revisões clínicas são definidas pela dualidade da medicina como uma ciência e uma arte. Elas precisam ser elaboradas de forma rigorosa, compatível com sua relevância, ao mesmo tempo em que exigem de seus autores experiência clínica para situar as evidências clínicas no contexto da assistência à saúde e redigir um artigo cuja leitura seja instrutiva. Trata-se essencialmente de transformar o processo de autoinstrução profissional através do cuidado redobrado em cada uma de suas etapas.

É interessante observar como as revisões clínicas compartilham características de diversos tipos de revisão de literatura. As revisões clínicas vão além da síntese de evidências, como as revisões críticas; têm o objetivo de informar a prática, como as revisões rápidas; concentram-se na literatura mais recente, como as revisões do estado da arte; e têm um escopo amplo e privilegiam as melhores evidências, como as pesquisas sistemáticas seguidas de revisão. A considerável sobreposição entre os diversos tipos de revisão de literatura é um fenômeno já conhecido, o que não significa que esses tipos sejam intercambiáveis. ${ }^{9}$

Esta proposta metodológica deve ser comparada a duas outras disponíveis na literatura. Na primeira proposta, o editor-chefe da AFP e colaboradores orientam a preparação de revisões clínicas para a própria revista. ${ }^{20}$ Apesar de muito daquela proposta continuar válido, alguns aspectos deixaram de ser favorecidos pela própria revista, que atualmente não cita o artigo em suas instruções aos autores. 
A outra proposta diz respeito às "revisões baseadas na evidência”, um tipo de revisão frequentemente confundindo com revisões clínicas. ${ }^{21}$ Assim como as revisões clínicas, as revisões baseadas na evidência incorporam tanto artigos originais quanto revisões sistemáticas e diretrizes clínicas ("normas de orientação clínica", em português europeu), e frequentemente avaliam a força das evidências com o SORT. No entanto, as revisões baseadas na evidência são consideradas uma forma especial de revisão sistemática e, como tais, têm perguntas bem definidas e relatam o percurso de obtenção das evidências. ${ }^{21}$ Cumpre mencionar ainda a proposta de Savassi et al. ${ }^{22}$ para a "busca de evidências em atenção primária à saúde", que traz instruções semelhantes para a resolver dúvidas clínicas mas não chega a desenvolver as demais etapas da elaboração de uma revisão clínica.

A medicina de família e comunidade no Brasil tem uma considerável produção de sumários de evidências clínicas, na forma de livros-texto baseados em evidências e programa de educação continuada. A produção de revisões clínicas, no entanto, não tem sido tão expressiva, apesar da recente ênfase pela RBMFC. ${ }^{23}$ Mesmo sem a pretensão de ser a última palavra sobre o assunto, espera-se que esta proposta metodológica seja útil para estimular e instrumentalizar a produção de mais e melhores revisões clínicas em medicina de família e comunidade em nosso país.

\section{Agradecimentos}

Este artigo não seria possível sem os (atuais e ex-) médicos residentes em medicina de família e comunidade da Universidade Vila Velha, que identificaram dificuldades na elaboração do trabalho de conclusão de curso e avaliaram a utilidade de versões preliminares deste artigo.

\section{Referências}

1. Sackett DL, Rosenberg WM, Gray JA, Haynes RB, Richardson WS. Evidence based medicine: what it is and what it isn't. BMJ. 1996;312(7023):71-2. https://doi.org/10.1136/bmj.312.7023.71

2. Djulbegovic B, Guyatt GH. Progress in evidence-based medicine: a quarter century on. Lancet. 2017;390(10092):415-23. https://doi. org/10.1016/S0140-6736(16)31592-6

3. Smith R, Rennie D. Evidence-based medicine—an oral history. JAMA. 2014;311(4):365-7. https://doi.org/10.1001/jama.2013.286182

4. Guyatt GH, Meade MO, Jaeschke RZ, Cook DJ, Haynes RB. Practitioners of evidence based care. Not all clinicians need to appraise evidence from scratch but all need some skills. BMJ. 2000;320(7240):954-5. https://doi.org/10.1136/bmj.320.7240.954

5. Ely JW, Osheroff JA, Ebell MH, Chambliss ML, Vinson DC, Stevermer JJ, et al. Obstacles to answering doctors' questions about patient care with evidence: qualitative study. BMJ. 2002;324(7339):710. https://doi.org/10.1136/bmj.324.7339.710

6. Green ML, Ruff TR. Why do residents fail to answer their clinical questions? A qualitative study of barriers to practicing evidence-based medicine. Acad Med. 2005;80(2):176-82. https://doi.org/10.1097/00001888-200502000-00016

7. Alper BS, Haynes RB. EBHC pyramid 5.0 for accessing preappraised evidence and guidance. Evid Based Med.2016;21(4):123-5. https:// doi.org/10.1136/ebmed-2016-110447

8. Institute of Medicine (U.S.). Clinical practice guidelines we can trust. Washington, DC: National Academies Press; 2011.

9. Grant MJ, Booth A. A typology of reviews: an analysis of 14 review types and associated methodologies. Health Inf Libr J.2009;26(2):91108. https://doi.org/10.1111/j.1471-1842.2009.00848.x

10. Haynes RB, Cotoi C, Holland J, Walters L, Wilczynski N, Jedraszewski D, et al.; McMaster Premium Literature Service (PLUS) Project. Second-order peer review of the medical literature for clinical practitioners. JAMA. 2006;295(15):1801-8. https://doi.org/10.1001/ jama.295.15.1801 
11. Vetter N. What is a clinical review? Rev Clin Gerontol. 2003;13(2):103-5. https://doi.org/10.1017/S0959259803013212

12. O'Connor D, Green S, Higgins JPT. Defining the review question and developing criteria for including studies. In: Higgins JPT, Green $\mathrm{S}$, Cochrane Collaboration, eds. Cochrane handbook for systematic reviews of interventions. Cochrane Book Series. Chichester (UK); Hoboken (USA): Wiley-Blackwell; 2008.

13. Ebell MH, Siwek J, Weiss BD, Woolf SH, Susman J, Ewigman B, et al. Strength of recommendation taxonomy (SORT): a patient-centered approach to grading evidence in the medical literature. J Am Board Fam Pract. 2004;17(1):59-67. https://doi.org/10.3122/jabfm.17.1.59

14. Guyatt GH, Oxman AD, Vist GE, Kunz R, Falck-YtterY, Alonso-Coello P, et al.; GRADE Working Group. GRADE: an emerging consensus on rating quality of evidence and strength of recommendations. BMJ. 2008;336(7650):924-6. https://doi.org/10.1136/bmj.39489.470347.AD

15. Guyatt GH, Oxman AD, Kunz R, Jaeschke R, Helfand M, Liberati A, et al.; GRADE Working Group Incorporating considerations of resources use into grading recommendations. BMJ. 2008;336(7654):1170-3. https://doi.org/10.1136/bmj.39504.506319.80

16. Guyatt GH, Oxman AD, Kunz R, Vist GE, Falck-Ytter Y, Schünemann HJ; GRADE Working Group. What is "quality of evidence" and why is it important to clinicians? BMJ. 2008;336(7651):995-8. https://doi.org/10.1136/bmj.39490.551019.BE

17. Brasil. Ministério da Saúde. Secretaria de Ciência, Tecnologia e Insumos Estratégicos. Departamento de Ciência e Tecnologia. Diretrizes metodológicas: Sistema GRADE - Manual de graduação da qualidade da evidência e força de recomendação para tomada de decisão em saúde. Brasília: Ministério da Saúde; 2014.

18. Guyatt GH, Oxman AD, Schünemann HJ, Tugwell P, Knottnerus A. GRADE guidelines: A new series of articles in the Journal of Clinical Epidemiology. J Clin Epidemiol. 2011;64(4):380-2. https://doi.org/10.1016/j.jclinepi.2010.09.011

19. Agoritsas T, Merglen A, Heen AF, Kristiansen A, Neumann I, Brito JP, et al. UpToDate adherence to GRADE criteria for strong recommendations: an analytical survey. BMJ Open. 2017;7(11):e018593. https://doi.org/10.1136/bmjopen-2017-018593

20. Siwek J, Gourlay ML, Slawson DC, Shaughnessy AF. How to Write an Evidence-Based Clinical Review Article. Am Fam Physician. 2002;65(2):251-8.

21. Braga R, Melo M. Como fazer uma revisão baseada na evidência. Rev Port Med Geral Fam. 2009;25(6):660-6.

22. Savassi LCM, Oliveira ACD, Dias RB. Proposta de metodologia de busca de evidências em Atenção Primária à Saúde: o exemplo da osteoporose em homens. Rev Bras Med Fam Comunidade. 2008;4(15):205-21. https://doi.org/10.5712/rbmfc4(15)177

23. Norman AH. A RBMFC e o desafio da educação médica continuada. Rev Bras Med Fam Comunidade. 2013;8(26):3-5. https://doi. org/10.5712/rbmfc8(26)719 